\title{
“Ponencia de Política Científica de la AE-IC" de 2020: un balance y algunas propuestas
}

The 2020 "Scientific Policy Presentation for AE-IC": a balance and some proposals for improvement

Marzal Felici, Javier

Universitat Jaume I (UJI)

marzal@uji.es

\section{García Galindo Juan Antonio}

Universidad de Málaga (UMA)

jagarciag@uma.es

Forma de citar este artículo:

Marzal Felici, J. y García Galindo, J. A. (2021). “Ponencia de Política Científica de la AE-IC" de 2020: un balance y algunas propuestas. RAE-IC, Revista de la Asociación Española de Investigación de la Comunicación, 8(15), 24-52. https://doi.org/10.24137/raeic.8.15.2

\section{Resumen:}

Se reproduce la "Ponencia de Política Científica", documento realizado por los profesores Javier Marzal Felici, Catedrático de Comunicación Audiovisual de la Universitat Jaume I de Castellón, y por Juan Antonio García Galindo, Catedrático de Periodismo de la Universidad de Málaga, por encargo de la Presidencia y de la Junta Directiva de la Asociación Española de Investigación de la Comunicación (AE-IC), y que 
ha sido redactado entre julio y octubre de 2020. Por un lado, el presente Informe, de carácter sintético y propositivo -esto es, "ejecutivo"-, ofrece un balance de la situación de la investigación en el campo de la comunicación en España, que trata de recoger numerosas reflexiones realizadas por varias decenas de investigadoras/es, socias y socios de la AE-IC. Por otro, la "Ponencia de Política Científica" se propone estimular incluso "provocar"- el debate interno en el seno de la AE-IC, en un momento en el que se percibe con nitidez que el campo científico de las ciencias de la comunicación ha alcanzado una notable madurez. Finalmente, los ponentes tratan de recoger las principales preocupaciones de una parte importante de la comunidad científica del campo de las ciencias de la comunicación, con el fin de construir futuros consensos, de la mayor amplitud posible, a través de la exposición de dieciséis recomendaciones sobre política científica.

Palabras clave: comunicación, investigación de la comunicación, política científica, evaluación de la investigación, epistemología, teoría del conocimiento.

\section{Abstract:}

Below is the Scientific Policy Presentation, a document created by Javier Marzal Felici, Professor of Audiovisual Communication at the Jaume I University of Castellón, and by Juan Antonio García Galindo, Professor of Journalism at the University of Málaga. It was commissioned by the Presidency and Governing Board of the Spanish Association of Communication Research (AE-IC) and written between July and October 2020. This report acts as a summary while making proposals; that is to say, it is "executive". On the one hand, it gives an overview of the situation for research in the field of communication in Spain, gathering numerous reflections given by several dozens of researchers and members of AE-IC. On the other, the Scientific Policy Presentation proposes to stimulate or even "provoke" internal debate within the heart of AE-IC at a time when it is clearly perceived that the sphere of communication sciences has reached a notable level of maturity. Finally, those presenting it are attempting to gather the main concerns of a large part of the scientific community in the field of communication sciences in order to build future consensuses that are as broad as possible by presenting sixteen recommendations on scientific policy. 
Keywords: communication, communication research, scientific policy, research evaluation, epistemology, theory of knowledge.

\section{REFLEXIÓN PRELIMINAR}

El presente Informe presenta un balance de la situación de la investigación en el campo de la comunicación, encargado por la Junta Directiva de la AE-IC, basado en la experiencia profesional de los ponentes firmantes de este Informe que, por tanto, contiene valoraciones inevitablemente subjetivas. Se trata de un breve análisis que intenta sintetizar numerosas reflexiones realizadas por varias decenas de investigadoras/es, socias y socios de la Asociación Española de Investigación de la Comunicación.

En primer lugar, creemos necesario subrayar el hecho de que nos hallamos ante una realidad académica de una enorme complejidad. Por ello resulta difícil, si no imposible, desligar la actividad investigadora de otras actividades que forman parte del trabajo cotidiano de los académicos, como la transferencia, la docencia y la gestión, con las que está íntimamente ligadas. En segundo lugar, el objetivo principal de este Informe es identificar las principales debilidades y carencias que presenta el sistema de investigación de la comunicación en España, así como realizar una serie de recomendaciones que pueden traducirse en líneas de acción a desarrollar en el futuro, con el fin de consolidar nuestro campo científico. En este sentido, reconocemos que nuestra principal preocupación es el desarrollo futuro de la investigación de la Comunicación en el contexto latinoamericano que, necesariamente, dependerá de las nuevas generaciones de investigadores que se irán incorporando a la AE-IC.

Asimismo, queremos manifestar nuestra deuda con el Informe realizado en 2013, Política científica y tecnológica de investigación en comunicación. Nuevas Bases de Organización del Campo Académico, por Francisco Sierra Caballero y José Luis Piñuel Raigada, así como con la actividad desarrollada por numerosos investigadores e investigadoras de la Sección "Teoría y Métodos de Investigación en Comunicación”, cuya 
producción científica constituye una fuente de inspiración permanente para la reflexión sobre la naturaleza de la investigación en comunicación.

También debemos destacar que desde 2013 hasta hoy el sistema de evaluación de la calidad de la producción científica ha sufrido una fuerte evolución, desde un modelo puramente cualitativo - muy criticado por su carácter bastante "arbitrario", a priori-, a un modelo esencialmente cuantitativo - que ignora muchos aspectos relevantes de la naturaleza de la producción científica- Nuestra perspectiva de trabajo se sitúa en la búsqueda de un punto de equilibrio, en el que sea posible compatibilizar ambas visiones que, a nuestro juicio, deben ser complementarias. En este sentido, sigue siendo referente el modelo mixto de evaluación científica que se practica en el mundo anglosajón.

En definitiva, el estilo sintético y directo del presente Informe, de naturaleza "ejecutiva", tiene como finalidad última estimular -incluso "provocar" - el debate interno en el seno de la $A E-I C$, en un momento en el que se percibe con nitidez que el campo científico de las ciencias de la comunicación ha alcanzado una notable madurez, si bien creemos que, para seguir avanzando, debemos ser muy críticos (y autocríticos) con la realidad académica que nos rodea. En suma, nuestra aspiración es recoger las preocupaciones de una parte importante de la comunidad científica del campo de las ciencias de la comunicación, para construir futuros consensos, con la mayor amplitud posible.

También queremos aprovechar esta nota preliminar para agradecer la confianza depositada y todo el apoyo recibido de la actual Junta Directiva de la AE-IC y, muy especialmente, de su presidente, Enrique Bustamante Ramírez.

Finalmente, pedimos disculpas por la omisión de muchas referencias a estudios e investigaciones que han aparecido en los últimos años. Cabe destacar la intensa actividad de nuestros/as asociados/as en torno a la llamada "metainvestigación" o epistemología de la comunicación, imprescindible para la maduración del campo científico. Las características de este Informe, que ha de ser necesariamente breve, nos ha obligado a reducir las referencias todo lo posible. 


\section{UNA VISIÓN PANORÁMICA DEL CAMPO CIENTÍFICO DE LA COMUNICACIÓN EN 2020}

En 2022, los estudios de comunicación en España cumplirán 50 años de vida. Son casi cinco décadas de intenso trabajo, que han llevado a la consolidación de un campo científico que no existía con anterioridad, de forma oficial en España.

No obstante, cabe preguntarse si la Comunicación es, en efecto, un campo científico perfectamente reconocible en 2020 entre el conjunto de saberes y ramas del conocimiento. Por el momento, seguimos sin tener una identidad reconocible en el marco de los llamados "códigos UNESCO", una nomenclatura internacional, que describe los campos de la Ciencia y Tecnología, establecida en 1973 y 1974 por las Divisiones de Política Científica y de Estadística de la Ciencia y Tecnología de la UNESCO, adoptada por el Ministerio de Ciencia y Tecnología de España en 1983, con pequeñas modificaciones introducidas en 1986 y 1988. Atendiendo a la máxima del filósofo George Steiner "lo que no se nombra, no existe" - en realidad, un principio clásico de la filosofía del lenguaje-, la Comunicación como "campo científico" no existe oficialmente en la actualidad, si no es subsumido de forma muy imprecisa e insatisfactoria en otros campos científicos como la historia (55), las ciencias económicas (53), la psicología (61), la física (22), las "ciencias de las artes y las letras" (62), la sociología (63), la pedagogía (58) y las “ciencias tecnológicas” (33).

Las propias denominaciones utilizadas por la UNESCO - casi medio siglo atrás - parecen reclamar una revisión en profundidad del listado de códigos, utilizados habitualmente para cumplimentar documentos tan comunes como la solicitud de proyectos de investigación, la solicitud y adscripción de becas de investigación, la clasificación de nuestra producción científica, etc. No es descabellado pensar que más tarde o temprano se procederá a revisar los "códigos UNESCO", por lo que consideramos urgente la realización de un estudio riguroso que permita fijar un mapa lo más detallado y completo posible del campo científico de las "ciencias de la comunicación". Dicho estudio serviría para identificar las "disciplinas" y "subdisciplinas" que se pueden determinar en los ámbitos del periodismo, de la comunicación audiovisual y de la publicidad y de las relaciones públicas. En el IV Congreso Internacional de la AE-IC, celebrado en Bilbao en 2014, fue presentado un análisis de la cuestión (Marzal-Felici, 
García-Jiménez y Humanes, 2016, pp. 65-79), aún hoy sin desarrollar, que debería concretarse en la propuesta de un nuevo campo científico, que reclaman muchos asociados y asociadas de la AE-IC (García García, 2007). En definitiva, es urgente avanzar en el diseño de un mapa de "la geografía gnoseológica del campo de la comunicación, qué tipos de espacios científicos se pueden identificar en su seno, y cómo se interrelacionan entre sí, más allá de la especialización de cada investigador, en [este] campo concreto del saber" (Marzal-Felici, García-Jiménez y Humanes, 2016, pp. 65-79), como territorio de cruce de saberes, muy fértil para el desarrollo de la interdisciplinariedad, en definitiva, de diálogo entre diferentes tradiciones de pensamiento - la semiótica, la teoría del discurso, la teoría crítica, la economía política de la comunicación, los estudios culturales, las aproximaciones sistémicas, etc. - , como propugnan distintos estudiosos (Craig, 1999, 2016; Bernardo Paniagua, 2006; GarcíaJiménez, 2016)

Actualmente, los estudios de comunicación están adscritos al ámbito de las ciencias sociales por instituciones oficiales como el Ministerio de Universidades, ANECA y las agencias de evaluación de calidad de las comunidades autónomas. Las áreas de conocimiento "Periodismo" y "Comunicación Audiovisual y Publicidad" (cuya conceptualización también merecería una revisión en profundidad) están actualmente adscritas en los diferentes programas de ANECA como Academia, PEP, CNEAI, Verifica, Acredita, Audit, etc., a la Rama de "ciencias sociales y jurídicas". Más específicamente, en el programa de evaluación del profesorado para las acreditaciones a CU y TU, las áreas de "Periodismo" y "Comunicación Audiovisual y Publicidad" aparecen adscritas a la Comisión D18 "Ciencias sociales", junto a las áreas de conocimiento "Antropología social", "Ciencia política y de la administración", "Documentación", "Filosofía Moral”, "Sociología" y "Trabajo social y servicios sociales".

Por otro lado, en la CNEAI, comisión que se ocupa de evaluar los sexenios de investigación, las áreas de conocimiento de "Periodismo" y "Comunicación Audiovisual y Publicidad" están adscritas al campo no 7, subcampo 7.1 “Ciencias Sociales, Políticas, del Comportamiento y de Estudios de Género". El nombre del campo científico "Comunicación", o el de las áreas de conocimiento, no aparece ni siquiera en la 
denominación de la Comisión de evaluación de los sexenios. Antes lo ha hecho "Estudios de Género", que ha sido incluida en la convocatoria de 2019 -lo que nos parece muy positivo, sin duda-, si bien sería de justicia que se incluya también la denominación del campo científico "Comunicación". No son pocos los asociados y asociadas que reclaman que Comunicación tenga un campo propio, tanto para la evaluación de las solicitudes para las acreditaciones a los cuerpos de funcionarios y para las figuras laborales, como para la evaluación de los sexenios de investigación. Esta reclamación nos parece especialmente pertinente cuando se constata que cerca de un $40 \%$ de las solicitudes para la acreditación de profesores, verificación de títulos o para el reconocimiento de sexenios, en el ámbito de las ciencias sociales, son realizadas por PDI perteneciente a las áreas de conocimiento de "Periodismo" y "Comunicación Audiovisual y Publicidad".

En este sentido, constatamos con preocupación que la Fundación Española de Ciencia y Tecnología (FECYT) haya unificado en el "Ranking de revistas con sello de calidad FECYT" a los campos "Comunicación" e "Información y Documentación Científica". Creemos que la asimilación de ambos ámbitos disciplinares demuestra un profundo desconocimiento de la compleja naturaleza de los objetos de estudio propios del campo “Comunicación”, así como la diversidad de tradiciones epistemológicas que abarca este ámbito, frente al campo "Información y Documentación Científica", que tiene unos intereses y perspectivas de trabajo muy diferentes. Consideramos que se trata de un error que debe corregirse lo antes posible.

A nuestro juicio, la principal motivación que justifica esta reclamación es reconocer la complejidad del campo científico Comunicación, ya que engloba saberes teóricos y prácticos, que convocan la utilización de metodologías y técnicas de investigación muy diversas. En este sentido, creemos que, desde otros ámbitos, supuestamente muy cercanos, no se entiende que el campo de estudios sobre la comunicación es un espacio (inter) disciplinar que se encuentra a caballo entre las humanidades y las ciencias sociales, que ha terminado construyendo una identidad propia. En efecto, las áreas de conocimiento de "Periodismo" y "Comunicación Audiovisual y Publicidad" acogen investigaciones teóricas y aplicadas en los ámbitos de los estudios de recepción, sobre marcas e identidad corporativa, comunicación estratégica, comunicación de crisis, 
economía política de la comunicación, comunicación política, estudios culturales, estudios de género, historia de la comunicación, análisis de los discursos audiovisuales, realización y producción audiovisual, tecnologías de la comunicación, géneros periodísticos, ciberperiodismo, etc., que demuestra la existencia una enorme variedad de temáticas y enfoques de estudio. En este sentido, sostenemos que en esta diversidad y pluralidad científica radica la relevancia y riqueza del campo científico de la comunicación, que es necesario reivindicar.

\section{NUEVAS PRÁCTICAS INVESTIGADORAS, NUEVOS DESEQUILIBRIOS}

En la última década destaca el aumento exponencial del número publicaciones científicas, un hecho indicativo del dinamismo de nuestro ámbito científico, que debe valorarse a priori de forma muy positiva.

No obstante, y en menor medida, también es conocida la existencia de malas prácticas científicas, como la "publicación duplicada" de mismos textos en diferentes formatos de publicación. Los expertos en documentación también han constatado, a nivel general, la existencia de otras prácticas perniciosas como la existencia de "revistas depredadoras" - que no siguen los principios éticos de la revisión científica-, la "asociación entre revistas" o de grupos de investigadores para "intercambiar" citas, el exceso de "autocitas", la concentración de citas en los "marcos teóricos" -en ocasiones, sin justificación ni fundamento-, la alteración de datos, encuestas, etc. Por fortuna, los procesos editoriales son cada vez más rigurosos, si bien debemos ser conscientes de que queda mucho camino por recorrer. La dirección de la AE-IC constata con preocupación la existencia puntual de prácticas de esta naturaleza, que quizás podrían minimizarse con cambios en el actual modelo, que es mejorable, aunque bajo la dependencia de los máximos responsables de la política científica española.

La fuerte presión por publicar, siguiendo la máxima anglosajona del "publish or perish", ha aumentado la presión por publicar entre los investigadores, una adaptación necesaria a los sistemas de evaluación de la carrera académica que se han impuesto, consecuencia de que en la actualidad sólo se utilizan criterios cuantitativos - cuánto y dónde se publica-, por encima de lo que se publica; esto es, de la calidad de la producción científica. Aunque en los actuales criterios en vigor, sí se contempla la 
realización de una evaluación cualitativa, que un sistema colapsado y deficiente no puede asumir. Es también bastante evidente que esto ha conducido a un aumento muy notable de la producción científica, que no siempre está acompañada de la necesaria atención a la calidad del trabajo científico. Esta presión afecta de un modo especial a los investigadores más jóvenes al inicio de su carrera académica, repercutiendo negativamente en la calidad de la investigación y en su propia formación científica.

Nos parece necesario recordar el origen de la actual "presión por publicar", en especial, en revistas internacionales. El Informe Estrategia española de Ciencia y Tecnología y de Innovación 2013-2020, publicado por el Ministerio de Economía y Competitividad en 2012, proponía como uno de sus seis ejes prioritarios "el apoyo a la Internacionalización y promoción del Liderazgo Internacional del Sistema Español de Ciencia, Tecnología e Innovación, ya que representan un claro factor de competitividad y diferenciación que es imprescindible potenciar" (Ministerio de Economía y competitividad, 2010, p. 6). Una de las claves que explica el desarrollo exponencial del artículo como formato de publicación científica en los últimos años es, precisamente, la necesidad de cumplir con la internacionalización de la producción científica española, para así proyectarla hacia el exterior, y ganar de este modo en visibilidad y competitividad. No obstante, llama la atención otro aspecto del Informe del Ministerio de Economía y Competitividad de 2012 que no puede pasar desapercibido: en ningún momento se contempla el establecimiento de diferencias entre ámbitos científicos -básicamente entre las humanidades y ciencias sociales frente a las ciencias naturales y experimentales-. En la práctica, esto corre el riesgo de entenderse como un modo de uniformizar el pensamiento científico. Este último asunto no es en absoluto menor, puesto que la manera de difundir, divulgar y compartir la ciencia también condiciona, de manera muy relevante, la forma de investigar, esto es, de definir, estructurar, argumentar y articular el pensamiento científico (Marzal, Soler y Rodríguez, 2020).

En primer lugar, y debido precisamente a la necesidad de publicar "mucho y rápido", se han ido imponiendo hábitos de trabajo basados en la utilización intensiva de internet, que está cambiando la forma de trabajar de académicos e investigadores. Cada vez hay más recursos en la red, lo que permite trabajar más deprisa y aumentar así la 
productividad científica, con el riesgo de obviar información relevante que, muchas veces, no está disponible en internet, sino en los centros de documentación. En efecto, las bibliotecas han percibido, de forma muy nítida, el impacto de internet, y progresivamente han ido constatando que los académicos consultan menos sus fondos documentales y bibliográficos, con la excepción de los repositorios digitales que han ido creando las bibliotecas de nuestras universidades. En este sentido, numerosos miembros de la AE-IC constatan que los investigadores disponemos cada vez de menos tiempo para estudiar e investigar, con un ritmo adecuado.

En segundo lugar, como vienen denunciando las editoras/es de revistas y colecciones de libros científicos, es inadmisible el plagio en el contexto académico, que ha alcanzado cuotas inimaginables hace pocos años, en el contexto de la nueva cultura de la "eresearch". Por fortuna, la utilización de programas antiplagio, aunque con un coste económico importante para universidades e instituciones, está sirviendo para frenar estas malas prácticas, más frecuente en los trabajos académicos de los estudiantes de grado y máster. Creemos más necesario que nunca la aplicación de los códigos éticos y las buenas prácticas científicas.

En tercer lugar, otra derivada de la expansión de la nueva "e-research", quizás una de las más interesantes, es que internet ha hecho posible el trabajo colaborativo entre investigadores de centros de investigación muy alejados geográficamente entre sí, gracias al Ilamado "e-clouding". Pero no es menos cierto que la presión por publicar ha distorsionado, en ocasiones, lo que debería entenderse por trabajo colaborativo, convirtiéndolo algunas veces en trabajo "cooperativo", con una división del trabajo que, muchas veces, persigue multiplicar el número de publicaciones.

Finalmente, hay que señalar que el sistema español de evaluación de la investigación y el modelo de incentivación de la producción científica no ayuda precisamente a corregir esta situación, sino que la agrava. La máxima "publish or perish" ha provocado el desarrollo de un contexto en el que las revistas científicas, muy bien adaptadas al nuevo entorno digital, compiten entre sí para atraer a autoras/es con elevados índices de citación, con el fin de subir en los rankings de revistas como el Journal Citation Report de Clarivate o Scopus de Elsevier. La citación de artículos publicados por la misma revista 
donde deseamos ver publicado nuestro artículo y la inclusión de referencias de artículos recientes son dos elementos bien valorados por las propias revistas, que ya no explicitan estos criterios en sus respectivas normativas, por tratarse de recomendaciones éticamente más que cuestionables. Nos hallamos, pues, ante un modelo de promoción de la investigación que desincentiva la consulta de libros, informes y otras fuentes documentales que, con frecuencia, se encuentran en otros circuitos de difusión, cuya accesibilidad no es tan alta, en especial en el caso de los libros académicos, que no siempre cuentan con una versión digital, ni suelen ser gratuitos.

\section{LA EVALUACIÓN DE LA ACTIVIDAD INVESTIGADORA EN ESPAÑA}

Existe un consenso bastante amplio a la hora de señalar que el actual modelo de evaluación de la actividad investigadora es una de principales causas que explican la situación actual de deterioro de la calidad de la producción científica en España, que no es exclusiva, ni mucho menos, de nuestro campo científico.

\subsection{LOS PERNICIOSOS EFECTOS DEL FACTOR DE IMPACTO DE LAS REVISTAS COMO CRITERIO PARA EVALUAR LA CALIDAD DE LA PRODUCCIÓN CIENTÍFICA}

En efecto, la utilización del "Factor de Impacto" (FI) de las revistas como el principal criterio para medir la calidad de la producción científica es, ciertamente, uno de los problemas más graves del modelo de investigación que se ha terminado imponiendo en nuestro país, como hemos denunciado numerosos investigadores (entre otros, Reig, 2014; Marzal, Rodríguez y Gil, 2018; Delgado López-Cózar y Martín-Martín, 2019). Como explican los profesores Emilio Delgado y Alberto Martín (2019), España es un país excepcional en lo que se refiere a la importancia que concede al FI, cuya concepción por Eugene Garfield no pretendía el uso que actualmente están haciendo las agencias de evaluación de calidad y numerosas universidades españolas. Desarrollado originalmente, en 1972, para orientar a las bibliotecas universitarias en la adquisición de las revistas más consultadas por la comunidad científica, se ha convertido en la regla de oro que ha terminado revolucionando el panorama de la ciencia, muy especialmente en los ámbitos de las ciencias sociales y de las humanidades. Recordemos, asimismo, que el "Factor de Impacto" se refiere a la revista donde se publica, no a un artículo científico concreto que se somete a evaluación: para calcular el Fl se toman en cuenta 
el número de citas recibidas en un año concreto de artículos publicados en los dos años anteriores, y se dividen por el total de artículos publicados en esos dos años. De este modo, se evalúa la calidad de cada artículo con un indicador genérico de la revista; esto es, se extrapola el éxito de la revista al del artículo, lo que es sencillamente una falacia.

Como explican Delgado y Martín-Martín (2019), el uso inadecuado del "Factor de Impacto" llevó a la European Association of Science Editors (EASE), sociedad científica que agrupa a los profesionales de la edición de revistas científicas, a posicionarse contra este modelo en 2007. Pocos años después, la American Society for Cell Biology (ASCB) lanzó en 2012 la conocida Declaración de San Francisco sobre la Evaluación de la Investigación (DORA), que se manifestó en contra del FI y, en 2015, un grupo muy nutrido de especialistas en bibliometría firmaron el Manifiesto Leiden, que también reclama un modelo de evaluación de la investigación más cualitativo.

En este sentido, los autores de esta ponencia consideran necesaria, incluso urgente, la adhesión de la Asociación Española de Investigación de la Comunicación (AE-IC) a la Declaración de San Francisco sobre la Evaluación de la Investigación (DORA), así como al Manifiesto de Leiden, que apunta en la misma dirección, como acción para visibilizar que el sistema de valoración de la investigación en España no puede basarse únicamente en los valores métricos que proporcionan los rankings y las bases de datos como el Journal Citation Reports (JCR) de Clarivate o Scopus de Elsevier / Scimago Journal \& Country Rank (SJR) de las revistas científicas que, de ningún modo, pueden sustituir las decisiones informadas. Como señala el Manifiesto de Leiden, siguiendo la explicación de Lluis Codina, conocido especialista en documentación científica, "los indicadores han proliferado: normalmente bien intencionados, no siempre bien informados, y a menudo mal aplicados. Cuando organizaciones sin conocimiento sobre buenas prácticas e interpretación apropiada de indicadores llevan a cabo las evaluaciones, corremos el riesgo de dañar el sistema científico con los mismos instrumentos diseñados para mejorarlas". El actual modelo de evaluación que sigue el programa Academia de ANECA para la acreditación a las figuras de Catedrático y Titular de Universidad en Ciencias Sociales (pero no sólo, incluso también las comisiones de la rama de "Arte y Humanidades"), o la Comisión de evaluación de los sexenios de investigación del campo 
no 7, subcampo 7.1 “Ciencias Sociales, Políticas, del Comportamiento y de Estudios de Género", es excesivamente rígido y mecanicista en su aplicación, ignorando la naturaleza cualitativa de la actividad investigadora, lo que nos aleja de los estándares internacionales de evaluación de la investigación.

Cabe señalar que en 2019, la Universitat Oberta de Catalunya (UOC) firmó la Declaración de San Francisco (DORA) y, más recientemente, la Plataforma de Revistas de Comunicación, PlatCom -integrada por 13 revistas del campo de la comunicación: Revista Mediterránea de Comunicación, Communication Papers, index comunicación, Fotocinema, Fonseca Journal of Communication, Miguel Hernández Communication Journal, TecCom Studies, Sphera Publica, IROCAMM, adComunica, Pangea, ZER y Revista Latina de Comunicación Social-, acordó el pasado 2 de octubre de 2020 la firma de la Declaración de San Francisco y del Manifiesto de Leiden.

\subsection{LA PREEMINENCIA DE LAS REVISTAS FRENTE A OTROS FORMATOS DE PUBLICACIÓN COMO EL LIBRO O LA MONOGRAFÍA CIENTÍFICA}

Así pues, el sistema de acreditación vigente presupone que en ciencias sociales las investigaciones publicadas como artículos tienen un impacto superior a los libros (de autoría individual o colectiva), utilizando baremos discutibles como el índice de citación. En áreas como "Periodismo" y "Comunicacion Audiovisual y Publicidad", y creemos que en la mayoría de ámbitos de las ramas de Ciencias Sociales y Jurídicas y de Arte y Humanidades, existe un consenso bastante amplio sobre la relevancia del libro como formato de publicación, cuyo impacto en la comunidad científica es bastante superior a los artículos publicados en revistas científicas. Esta afirmación ha sido avalada por numerosos estudios e investigaciones (entre otros, Giménez, 2016; Muller, 2018; Rodríguez y Gil, 2018). Al mismo tiempo, es necesario distinguir entre el libro, monografía o ensayo de investigación y el libro o manual docente que, como es lógico, no cabe inscribir dentro de esta categoría de investigación.

Desde hace casi una década, se viene observando desde la dirección de la AE-IC, que los jóvenes investigadores ya no publican libros, ni desean publicar sus comunicaciones en los libros de actas que editan las sociedades científicas, que se reservan así para la publicación en revistas indexadas en JCR o Scopus. Esta realidad que perciben con 
claridad nuestros jóvenes investigadores también provoca su desafección y baja participación en las actividades que se impulsan desde las asociaciones científicas, lo que tiene graves consecuencias para el propio desarrollo de nuestras disciplinas. La reivindicación del libro como formato de publicación científica se justifica, sobre todo, porque la investigación en ciencias sociales y humanidades no responde en exclusiva a los métodos y formatos de las ciencias naturales o experimentales, cuyo medio natural es la revista. Cabe subrayar que, en estos ámbitos, no siempre es factible sintetizar en 5.000 / 8.000 palabras investigaciones de gran complejidad humanística y social que requieren -como marca el sentido común- de la extensión, tiempo y modo de lectura que ofrece el libro, frente al artículo para una revista. Si esto no se tiene en cuenta, se potencia una investigación, muchas veces de corto alcance, simplificada en ocasiones, sometida a los temas de moda ("fácilmente publicables") y con un rendimiento basado en la bonificación inmediata ("mayor número de citas que haga aumentar, a la máxima velocidad, el índice $h$ del investigador/a"), olvidando que en nuestras áreas de conocimiento es esencial el desarrollo de un pensamiento complejo y de la reflexión crítica, que un formato de publicación como la revista científica no puede facilitar al mismo nivel que el libro. No obstante, con esta reflexión no se está reclamando que se deba abrazar al libro académico para renunciar a la publicación de artículos en "revistas científicas": muy al contrario, se trata de dos formatos de publicación complementarios, y absolutamente necesarios, sin menoscabo de otros formatos de publicación existentes.

Por otra parte, queremos manifestar que tomar en consideración para la acreditación a Catedrático/a de Universidad únicamente la publicación de artículos en revistas indexadas en el Journal Citation Reports (JCR) C1 y C2 de Clarivate (Social Sciences Citation Index) y en C1 de SJR / Scopus, correspondientes al llamado nivel 1, también supone un cuestionamiento de la calidad de la producción científica española que publican nuestras revistas (que tienen una presencia muy reducida en esas bases de datos). Para CU, las revistas de nivel 2 incluyen: C3 y C4 del JCR, C2 y C3 del SJR / Scopus, C1 de Dialnet Métricas y C1 y C2 de FECYT. Para la acreditación a Titular de Universidad, la exigencia es un poco más "laxa": en el nivel 1 se contemplan C1 y C2 de JCR y C1 del SJR / Scopus; en el nivel 2, C3 y C4 del JCR, C2 y C3 de SJR / Scopus, C1 y C2 de Dianet 
Métricas y C1, C2 y C3 de FECYT. En este sentido cabe recordar que mientras que en JCR en Comunicación apenas hay 90 revistas, de las cuales 42 son C1 y C2; en Economía y Empresa se pueden contabilizar 874 revistas, de las cuales 437 corresponden a los cuartiles C1 y C2; y en Psicología existen en JCR 679 revistas, de las que 340 se encuentran en $\mathrm{C} 1$ y $\mathrm{C} 2$. Es evidente que la comunidad científica de Comunicación, que ha crecido en las últimas décadas de manera muy notable, está en inferioridad de condiciones con respecto a otros campos de saber.

Es necesario recordar que la gran mayoría de revistas que forman parte de las bases de datos de Clarivate y Elsevier no son de acceso abierto, y que buena parte de las investigaciones que publican es gracias a fondos públicos. Desde la economía del conocimiento, es fundamental tener en cuenta que, aunque las bases de datos de las multinacionales referidas son internacionales y están ampliamente aceptadas, sin embargo, ocultan un fabuloso negocio (publicación de investigaciones que no financian; autores que, no sólo no cobran por lo que publican, sino que pagan por publicar, mediante tasas y suscripciones a las revistas; revisores ciegos que no cobran; etc.) que, cuanto menos, contradice el desarrollo de políticas públicas europeas como el acceso a la "ciencia abierta (open access)". Celebramos que, en los nuevos criterios para las acreditaciones, en vigor desde el pasado 15 de enero de 2020, se hayan incluido otras bases de datos como el sello de calidad para las revistas FECYT y la nueva base de datos de Dialnet Métricas, en sus niveles $\mathrm{C} 1$ y $\mathrm{C} 2$, aunque nos parece insuficiente. En el caso de las carreras académicas de trayectoria dilatada, los actuales criterios no contemplan otras bases de datos como IN-RECS, que ofrece información sobre el índice de impacto de las revistas para el ámbito de las ciencias sociales y las humanidades entre 1996 y 2009, un elemento que podría ser tenido en cuenta.

En efecto, nos parece una grave contradicción apelar al prestigio y relevancia del Journal Citation Reports de Social Sciences Citation Index de Web of Science (WoS), y dejar fuera otras bases de datos como "Arts \& Humanities Citation Index", también de Clarivate Analytics, que asimismo están incluidas en WoS, por el hecho de que no están jerarquizadas en cuartiles, ya que no están ordenadas por el "Factor de Impacto". La base de datos "Arts \& Humanities Citation Index", creada en 1975, recoge la actividad 
de 1.850 revistas científicas, en campos como "cine, radio y televisión" o "estudios culturales", de gran relevancia para las áreas de "Periodismo" y "Comunicacion Audiovisual y Publicidad" - se pueden contabilizar hasta 20 , las revistas incluidas en $A \& \mathrm{HCl}$ que tratan temas relacionados con la comunicación ${ }^{1}-$. Sin embargo, hasta hace poco tiempo (en los criterios del Programa Academia de 2017) sí se tomaba en consideración una base de datos como "Emerging Sources Citation Index" (ESCI), incluida en WoS por Clarivate en 2016, por su prestigio en la comunidad científica. También se suele perder de vista la importancia que tiene, en especial en algunos ámbitos de nuestro campo científico, la publicación de resultados de investigación en revistas profesionales, de reconocido prestigio, cuyo enfoque es marcadamente distinto al de las revistas académicas, pero que tienen un valioso impacto en las profesiones de la comunicación y para el mundo empresarial.

Por otra parte, no se puede ignorar que el ámbito de la edición del libro tampoco está libre de dificultades y problemas. Por un lado, es importante que los procesos de edición de libros sigan unos protocolos de trabajo rigurosos y transparentes, equivalentes a los que numerosas revistas científicas de nuestro campo han desarrollado. En este sentido, se considera un avance importante la creación del SPI (Scholarly Publishers Indicators), una serie de rankings de editoriales (general, por disciplinas, de editoriales extranjeras y de editoriales españolas) basado en la opinión de expertos españoles en ciencias sociales y humanidades, por parte del Grupo ILIA del CSIC, así como la creación del sello "Calidad en Edición Académica CEA-APQ", promovido por la Unión de Editoriales Universitarias Españolas (UNE), y avalado por FECYT y ANECA. No obstante, hay que señalar que buena parte de las editoriales españolas no pueden hacer frente a los costes económicos que tiene, en estos momentos, la acreditación de cada colección de libros, cuya validez es muy limitada en el tiempo - como ocurre también con las indexaciones y sellos de calidad de las revistas-. Es conveniente avanzar en la mejora de la calidad de la edición científica y, en un momento de generalización de portales de transparencia

1 Entre las revistas relacionadas con el campo de estudios de "comunicación", destacan: Canadian Journal of Film Studies - Revue Canadienne d'Études Cinematografiques, Communication and Critical-Cultural Studies, Film Comment, Film Criticism, Film Quarterly, Historical Journal of Film, Radio and Television, Historia y comunicación social, History of Photography, Hrvatski Filmski Ljetopis, Information and Culture, JCMS - Journal of Cinema and Media Studies, Journal of British Cinema and Television, Journal of Chinese Cinemas, Journal of Film and Video, Journal of Popular Film and Television, L'Atalante. Revista de Estudios Cinematográficos, Millennium Film Journal, Photography and Culture, Sight and Sound y Studies in French Cinema, de las cuales 2 son españolas. 
en todas las administraciones públicas, la dirección de la AE-IC considera oportuna la publicación periódica de los balances económicos de las revistas y editoriales, que reciben financiación pública.

Finalmente, la AE-IC desea dejar constancia de su reconocimiento a la labor que han venido realizando los editores de las revistas de comunicación, muy especialmente en la última década, cuyo trabajo ha sido y es fundamental para la consolidación del campo científico de las ciencias de la comunicación. También quiere extender estos agradecimientos a los editores/as de libros, a los/as directores/as de colecciones de libros, a las editoriales, a las instituciones públicas y privadas que publican y editan investigaciones y estudios sobre comunicación. En este sentido, se subraya la importancia de la existencia de múltiples formatos de publicación que, sin lugar a dudas, enriquecen el campo científico y contribuyen a expandir la diversidad y pluralidad de la investigación en comunicación, unos valores que deben cuidarse muy especialmente en tiempos de "monocultivo" en las publicaciones y de "pensamiento único". Entre otras cosas, porque esos formatos y soportes de publicación también forman parte del amplio espacio de la comunicación. El mundo editorial es, por su propia naturaleza, y en toda su extensión, objeto también de estudio de la comunicación y de la cultura. Razones a nuestro entender que debe obligar a la revisión de los formatos de publicación entre los criterios de evaluación de la investigación en comunicación.

\section{ESTANDARIZACIÓN CIENTÍFICA E INVESTIGACIÓN “MAINSTREAM": RIESGOS Y}

\section{AMENAZAS}

Ya se ha hecho referencia en este Informe a la importancia de la diversidad de la producción científica. Son muchos los asociados y asociadas de la AE-IC que perciben que en el campo de la investigación en comunicación se está imponiendo una manera de hacer ciencia cada vez más estandarizada, en general poco permeable a la innovación, y que responde en muchos casos a la consolidación e institucionalización de un canon investigador único, de marcado carácter funcionalista e instrumental. No son pocos los asociados y asociadas que muestran su preocupación por la multiplicación de investigaciones que priman los aspectos metodológico-procedimentales sobre la reflexión teórica, con una enorme fuerza (auto)replicante, basada en el empirismo 
cuantitativo que, con frecuencia, termina cultivando la tautología, la irrelevancia y la descripción mecánica, dejando así de lado la comprensión, la evaluación o, incluso, la intervención en el estudio de los fenómenos comunicativos, fuera de los intereses de la agenda investigadora (Carrasco-Campos y Saperas Lapiedra, 2016; Piñuel y otros, 2017; Goyanes, 2017; Díaz Nosty y de Frutos, 2017). Este tipo de investigación constituye una suerte de "mainstream", que está imponiendo modas, no sólo en el tipo de investigaciones que se realizan, sino también en las metodologías y técnicas de investigación que utilizan. La Junta Directiva de la AE-IC ha expresado, de forma reiterada, su preocupación por la escasez de investigaciones y de "ventanas de publicación" que aborden y permitan la publicación de temas críticos, complejos e incómodos, un tipo de aproximaciones, de carácter ensayístico que, en ocasiones, también enriquecen y aportan una "rentabilidad social", que nuestro entorno socioeconómico, profesional y cultural demanda.

En este sentido, una de las principales consecuencias de la obsesión por la internacionalización de la producción científica por parte de las administraciones españolas es la publicación en inglés de los artículos para las revistas científicas. Sin duda, la publicación en inglés, con el objetivo de difundir los resultados de la investigación es muy importante, y está fuera de discusión. No obstante, hay tres aspectos que suscitan, cuanto menos, algunas dudas. En primer lugar, debemos ser conscientes que, en los campos de las humanidades y de las ciencias sociales (a diferencia de los que ocurre en el campo de las ciencias naturales y experimentales), la publicación en inglés - de inglés académico, y de calidad - tiene un coste para las arcas públicas (y privadas) bastante considerable, con resultados no siempre generadores de gran impacto en la comunidad científica, según la propia FECYT. En segundo lugar, en ciencias sociales y humanidades, hacer "ciencia" en inglés tiene consecuencias incluso en lo que se investiga y cómo se investiga: se tratan temas de interés transnacional, lo que implica con frecuencia descuidar el estudio de problemáticas que tienen que ver con la comunicación local, y se suelen utilizar técnicas de investigación del gusto editorial de las revistas. En tercer lugar, se olvida con frecuencia que somos una comunidad de 500 millones de hispanoparlantes, y hacer ciencia en español es una forma de visibilizar la relevancia y de poner en valor la cultura y la investigación 
latinoamericana. Todavía está pendiente la construcción de un verdadero espacio académico latinoamericano, con el apoyo de las instituciones públicas españolas. Finalmente, no se puede olvidar la importancia que tiene hacer ciencia también en otras lenguas minoritarias -euskera, catalán y gallego-, un aspecto que es sistemáticamente ignorado por las agencias de evaluación de calidad, cuando se evalúa la calidad de la producción científica.

Como consecuencia, asimismo, de la estandarización de la investigación, dirigida casi únicamente a la publicación de artículos en revistas, la AE-IC quiere manifestar su preocupación por la dramática pérdida de relevancia de los congresos y encuentros científicos que, con mucho esfuerzo, organizan las sociedades y asociaciones científicas. Hay que recordar que este tipo de actividades son muy importantes para la creación de una comunidad científica y para visibilizar la actividad que realizan los investigadores en comunicación. Dada su relevancia estratégica para la difusión del conocimiento, las agencias de evaluación de calidad deben ponderar adecuadamente la relevancia de este tipo de actividades.

En los últimos años, como consecuencia de la crisis económica de 2008, se ha constatado una significativa disminución de recursos dedicados a la financiación de proyectos de investigación por el Ministerio y las administraciones públicas y privadas. También en este caso, la evaluación de los proyectos de investigación pasa por expertos en ciencias sociales, cuyo máximo responsable no suele ser especialista del campo "Comunicación". Desde la $\mathrm{AE}-\mathrm{IC}$, como han hecho otras sociedades científicas, se reclama una mayor sensibilidad de los responsables de la Agencia Estatal de Investigación hacia la idiosincrasia del campo de la comunicación, y la necesidad de valorar los resultados de investigación, no sólo en el número de artículos publicados en revistas indexadas en JCR o Scopus, sino también en la transferencia real que las investigaciones proyectan en los sectores productivos de la comunicación, en el campo educativo y en la sociedad en su conjunto. Asimismo, la AE-IC considera necesario aumentar las partidas dedicadas a la financiación de proyectos de investigación tanto de la AEI como de las administraciones autonómicas, que tendrá efectos muy positivos en economía, la 
sociedad y la cultura de nuestro país. Porque no se puede olvidar que la comunicación es uno de los pilares básicos de una sociedad moderna.

En este sentido, nos parece pertinente señalar que es necesario impulsar las investigaciones en el campo de los estudios de género, en relación específicamente con el ámbito de las ciencias de la comunicación. La promoción de la perspectiva de género en la investigación de la comunicación, así como la atención a la existencia de paridad en los órganos de gestión de sociedades científicas como la AE-IC y los organismos e instituciones relacionadas con la actividad investigadora, nos parece absolutamente prioritaria actualmente.

La adaptación de los investigadores al modelo de evaluación de la calidad de la investigación, vigente en estos momentos, ha convertido a las redes sociales académicas como Academia o Researchgate - con claras diferencias entre ambas-, entre otras, en un elemento esencial para la difusión de las investigaciones. En general, se trata de plataformas que tienen un claro afán de lucro, y que viven del trabajo que realizan los millones de investigadores-usuarios que las utilizan, aunque se presenten a sí mismas como "plataformas open Access", libres de intereses económicos. La fuerte presión por publicar también está alimentando en la comunidad científica, a título individual e institucional, un deseo irrefrenable por incrementar la reputación profesional, que puede conducir en algunos casos a la aparición de ansiedad y estrés entre los investigadores (Goyanes y Rodríguez-Gómez, 2018). Los profesores Delgado LópezCózar y Martín-Martín identifican la aparición de una nueva patología, muy creciente en la comunidad académica: la emergencia de la "ludopatía bibliométrica", o "impactitis", que está generando una suerte de "adicción a los números que etiquetan nuestra estima y reputación":

Todas las métricas de base bibliométrica con la citación a la cabeza poseen un fuerte componente narcisista que excitan nuestro ego sin límites. La continua recepción de alertas y mensajes de editores, redes sociales, buscadores, bases de datos generan inyecciones continuas de dopamina que inundan nuestros cerebros y que pueden conducir a graves adicciones (Orduna-Malea, Martín-Martín, Delgado López-Cózar, 2017, p. 14). 
Finalmente, consideramos que es urgente recuperar las preguntas básicas que cualquiera se plantea cuando aborda una investigación: “¿Qué temas son relevantes para el campo científico de la comunicación?”, "¿Por qué investigamos unos temas y no otros?" y “¿Qué impacto generará nuestra investigación, no sólo en la comunidad académica, sino en los sectores profesionales de la comunicación y en la sociedad en su conjunto?". Pueden parecer preguntas muy elementales, pero consideramos que son esenciales en la actualidad. La vorágine de publicaciones y actividades investigadoras nos impide, en ocasiones, reconocer qué temáticas son realmente relevantes y cómo pueden contribuir de forma positiva a nuestra sociedad. En este sentido, creemos que el impacto de la investigación científica sobre la sociedad ha de ser considerado no solo en términos de contribución al avance científico sino también en términos de utilidad social. A nuestro entender se impone la necesidad de medir dicho impacto y de establecer métricas relacionadas con la aplicación de la ciencia.

\section{LA RECLAMACIÓN DE UN NUEVO MODELO DE CARRERA UNIVERSITARIA, MÁS ABIERTO Y PLURAL}

Numerosos asociados y asociadas de la $\mathrm{AE}-\mathrm{IC}$ vienen expresando, en los últimos años, su malestar ante el hecho de que la evaluación de sus trayectorias académicas en los procesos de acreditación no tiene en cuenta la complejidad de las áreas de conocimiento "Periodismo" y "Comunicación Audiovisual y Publicidad", donde existe una gran variedad de perfiles investigadores y docentes que se sitúan a caballo entre las humanidades y las ciencias sociales.

En este sentido, muchas voces reclaman que las creaciones artísticas y las actividades profesionales sean valoradas adecuadamente, porque existen muchos perfiles académicos que sólo pueden alcanzar un alto nivel de excelencia si y sólo si los académicos han desarrollado una alta actividad creativa y profesional, y si mantienen un contacto directo con los sectores profesionales de la comunicación.

Esto nos lleva a señalar, aunque sea de forma sólo expositiva, que el problema de fondo es haber definido en España un único modelo de carrera académica, "monocromático", que sólo tiene en cuenta la excelencia investigadora, eso sí sobre unos parámetros muy discutibles, como hemos visto. Quizás sería conveniente desarrollar un nuevo modelo 
de carrera académica, más abierto y plural, que contemple la posibilidad de desarrollar distintos itinerarios de carrera académica, que permita aprovechar el trabajo que desarrollan muchos académicos, no sólo en el campo de la investigación, sino también en la transferencia, en la docencia o en la gestión.

\section{LA APUESTA POR LA "CIENCIA ABIERTA": HACIA UNA NUEVA CONCEPCIÓN DE LA ACTIVIDAD INVESTIGADORA}

En estos últimos años ha ido cobrando importancia el concepto de "ciencia abierta", un movimiento que fomenta que la investigación científica, las metodologías, técnicas de investigación, datos y resultados de las investigaciones, con el fin de que sean compartidos entre los miembros de la comunidad científica, sean reutilizables y accesibles al conjunto de la sociedad, de forma libre y gratuita. El programa Horizonte 2020 de la Comisión Europea declaraba en 2017 que "la ciencia abierta" es también una respuesta a la "demanda para abordar los cambios sociales de nuestro tiempo", que ha de contribuir al incremento de "la competitividad y el empleo", al fomentar la participación y colaboración entre científicos desde una perspectiva internacional.

El Center for Open Science (COS) señala que un aspecto central para el desarrollo de la ciencia abierta es la reconceptualización de la difusión y evaluación de la investigación, para lo que es necesario promover el trabajo colaborativo. Como se señala en el portal del Proyecto Foster Open Science, la ciencia abierta es mucho más que la disponibilidad en acceso abierto de publicaciones y datos: la ciencia abierta representa una apertura del proceso científico, que persigue reforzar la Responsabilidad Social Científica (RSC), que en última instancia se propone favorecer "Ia traslación del conocimiento científico a la sociedad". En realidad, la ciencia abierta representa un cambio revolucionario en la forma misma de entender y practicar la actividad investigadora. Por ello, creemos que, en una sociedad cada vez más interconectada, en la que nuestros datos son cada vez más públicos, es muy importante apostar por la "publicación en abierto" y por la "transparencia". Cabe reconocer que muchas revistas y colecciones de libros están desarrollando desde hace años este modelo de ciencia abierta, gracias al esfuerzo personal y económico de numerosos/as investigadores/as. Desde la Comisión de Política Científica de la AE-IC se emplaza a sus asociados y asociadas a que realicen un esfuerzo 
para publicar y difundir sus investigaciones en los repositorios de las universidades y en las redes académicas, con el fin de facilitar el acceso de la sociedad al conocimiento científico del campo de la comunicación. También es importante, en este contexto, que los investigadores/as revisen y corrijan los errores que detecten en sus perfiles de "Google Scholar", puesto que esta es la base de datos que recoge la variedad más amplia de tipos de publicaciones. Una acción de estas características permitiría ofrecer a la comunidad académica y a la sociedad una imagen más nítida y exacta del campo de estudios de la comunicación.

A juicio de los ponentes de este Informe, la ciencia abierta necesita la creación de un entorno de "slow research", parafraseando y transponiendo la afortunada expresión acuñada por Maggie Berg y Barbara K. Seeber en su obra The Slow Professor (2016), que cabe inscribir en el movimiento de la slow science (Pels, 2003). El desarrollo de investigación de la comunicación, de calidad, necesita de la inversión de tiempo para reflexionar. En definitiva, es imperativo pensar más en la calidad que en la cantidad de la producción científica. De este modo, la apelación al modelo de ciencia abierta constituye una reivindicación de otra manera de entender y abordar la investigación, de una nueva actitud ante el conocimiento, más necesaria que nunca.

\section{RESUMEN DE RECOMENDACIONES}

1. Es urgente la realización de un estudio riguroso que permita fijar un mapa lo más detallado y completo posibles del campo científico de las "ciencias de la comunicación", que identifique las disciplinas y subdisciplinas que se pueden determinar en los ámbitos del periodismo, de la comunicación audiovisual y de la publicidad y de las relaciones públicas, con el fin de reclamar la creación de un campo propio Comunicación o "ciencias de la comunicación" en el catálogo de los códigos UNESCO.

2. El Ministerio de Universidades, ANECA y otras agencias de evaluación de calidad deben crear comisiones propias de Comunicación para los procesos de acreditación, de evaluación de tramos de investigación, de acreditación o verificación de títulos, etc., como reconocimiento público a la especificidad de 
este campo científico, con la inclusión de la denominación "Comunicación" o "ciencias de la comunicación" en el nombre de dichas comisiones.

3. La comunicación constituye un campo científico con identidad propia, que ha configurado un espacio disciplinar específico, que se encuentra a caballo entre las humanidades y las ciencias sociales, con una clara vocación interdisciplinar. En la diversidad y pluralidad de objetos de estudio y de enfoques de investigación radica la relevancia y riqueza del campo científico de la comunicación, que debemos reivindicar. Por ello, la AE-IC considera necesario:

- "Comunicación" debe tener un campo propio entre las Comisiones de evaluación de los sexenios de CNEAI

- Solicitar a FECYT que "Comunicación" tenga un campo propio diferenciado de "Información y Documentación Científica", cuya tradición científica está muy alejada de los intereses, enfoques y metodologías de las investigaciones sobre periodismo y sobre comunicación audiovisual y publicidad.

4. Un análisis de las prácticas investigadoras del mundo académico actual, marcado por una fuerte presión por publicar, permite detectar un fuerte crecimiento de producción científica, en ocasiones redundante, que trata de dar respuesta a las exigencias del modelo vigente. La AE-IC advierte contra la producción científica replicante, contra el fraude, el plagio y la "depredación" de publicaciones y contra un trabajo falsamente "cooperativo", de división de funciones para sumar más "méritos", en lugar del trabajo "colaborativo", fruto del debate maduro y de un auténtico trabajo en equipo.

5. La AE-IC anuncia su adhesión a la Declaración de San Francisco sobre la Evaluación de la Investigación (DORA), de 2012, así como al Manifiesto de Leiden, de 2015, al considerar inadecuado el modelo de evaluación de la calidad de la producción científica que el programa Academia y la Comisión del campo 7 de la CNEAI, ambos dependientes de ANECA, siguen en estos momentos, porque las 
métricas no pueden substituir las decisiones informadas. Con el fin de corregir este modelo, la AE-IC propone:

- Programar un diálogo, permanente y estratégico, con la dirección de ANECA para trasladarle la realidad del campo.

- Elaborar un documento que sugiera ajustes en los actuales criterios del programa Academia y CNEAl y elevarlo a ambas comisiones de evaluación, en un plazo no superior a 6 meses. Dicho documento será resultado de un proceso participativo entre las asociadas/os de AE-IC.

- Crear y financiar un observatorio que monitorice la actividad investigadora del campo y proporcione informes periódicos con indicadores específicos del campo que sirvan para tomar decisiones motivadas en las políticas de evaluación.

- Impulsar de forma decidida una estrategia de grupo de presión que permita dar a conocer la realidad de AE-IC y del campo de la Comunicación a los principales públicos de interés.

- Desarrollar acciones para conocer y buscar convergencias con los modelos de evaluación de la investigación que se siguen en el sur de Europa -entre otros, con Francia, Italia y Portugal- y en el espacio latinoamericano.

6. La AE-IC solicita a las comisiones de evaluación de la calidad de la investigación del programa Academia de ANECA y la Comisión del campo 7 de la Comisión Nacional de Evaluación de la Calidad Investigadora (CNEAI) que tomen en consideración la inclusión de bases de datos como "Arts \& Humanities Citation Index" y "Emerging Sources Citation Index" (ESCI), que están reconocidas por una amplia mayoría de la comunidad científica del campo de la comunicación.

7. La AE-IC manifiesta el reconocimiento de la comunidad científica al trabajo que realizan los editores y editoras de las revistas de comunicación, así como a la labor de los editores/as de libros, a los/as directores/as de colecciones de libros, 
a las editoriales, a las instituciones públicas y privadas que publican y editan investigaciones y estudios sobre comunicación. La existencia de una amplia variedad de formatos y "ventanas" de publicación es esencial para el futuro de la investigación de la comunicación.

8. La AE-IC advierte del peligro de la estandarización de la investigación científica, de la necesidad de apostar por el estudio de temáticas variadas, de no descuidar la investigación de temas locales, de emplear metodologías y técnicas de investigación diversas y variadas, para dar respuesta a los retos y a la complejidad de la comunicación en el mundo actual.

9. La AE-IC reclama la importancia de hacer ciencia en español, así como el uso de las lenguas Ilamadas minoritarias - euskera, catalán y gallego-, sin menoscabo de la utilización del inglés como lengua franca en la comunicación científica.

10. La AE-IC constata con preocupación la pérdida de relevancia de los congresos y encuentros de investigadores que organizan las sociedades y asociaciones científicas, y reclama que las agencias de evaluación de calidad valoren la importancia de este tipo de actividades científicas.

11. La AE-IC reclama la necesidad de que se aumenten significativamente los presupuestos dedicados a la investigación en comunicación, por parte de la Agencia Estatal de Investigación y de las administraciones autonómicas, y que la evaluación de los resultados de investigación tenga en cuenta la transferencia real que las investigaciones proyectan en los sectores productivos de la comunicación, en el campo educativo y en la sociedad en su conjunto.

12. La AE-IC afirma la necesidad de promover la perspectiva de género en la investigación de la comunicación, así como la atención a la existencia de paridad en los órganos de gestión de sociedades científicas como la AE-IC y de los organismos e instituciones relacionadas con la actividad investigadora.

13. La AE-IC advierte contra los efectos negativos del uso inadecuado de las redes sociales académicas y anima a estudiar este fenómeno, que tan relacionado está con la creación de marca académica personal. 
14. La AE-IC emplaza a sus asociados y asociadas a reflexionar sobre el sentido de sus investigaciones, a apostar por la innovación y a redoblar esfuerzos para que los resultados de las investigaciones tengan un impacto real en los ámbitos profesionales de la comunicación, así como en la educación, en la cultura y la sociedad.

15. La AE-IC solicita a los responsables de las administraciones de la educación superior, a nivel estatal y autonómico, a explorar alternativas al actual modelo de carrera profesional de los académicos, que tenga en cuenta, no sólo la investigación, sino también otras dimensiones de la realidad del PDI como la transferencia, la excelencia docente y la gestión universitarias.

16. La AE-IC apuesta por el modelo de "ciencia abierta", por el acceso libre a las publicaciones científicas, el trabajo colaborativo y la transparencia en la investigación, y emplaza a sus asociados y asociadas a publicar sus investigaciones en los repositorios de las universidades y en las redes sociales académicas, así como a revisar y corregir los datos existentes en "Google Scholar", para ofrecer a la comunidad académica y a la sociedad una imagen más nítida y exacta del campo de estudios de la comunicación.

\section{REFERENCIAS BIBLIOGRÁFICAS}

Berg, M. y Seeber, B. K. (2016). The Slow Professor: Challenging the Culture of Speed in the Academy. Toronto: University of Toronto Press.

Bernardo Paniagua, J. M. (2006). El sistema de la comunicación mediática. De la comunicación interpersonal a la comunicación global. Valencia: Tirant Lo Blanch.

Carrasco-Campos, Á. y Saperas Lapiedra, E. (2016). Cambio tecnológico, globalización neoliberal y hegemonías metodológicas en la investigación comunicativa internacional. Revista Ámbitos, 32, cuarto trimestre (invierno). Recuperado de https://institucionales.us.es/ambitos/cambio-tecnologico-globalizacion-neoliberal-yhegemonias-metodologicas-en-la-investigacion-comunicativa-internacional/ 
Craig, R. T. (1999). Communication Theory as a Field. Communication Theory, 9(2), 119-161.

Delgado López-Cózar, E., Martín-Martín, A. (2020). El factor de impacto de las revistas científicas sigue siendo ese número que devora la ciencia española: ¿hasta cuando? Preprint. Anuario Think EPI, 14, e14e01. https://doi.org/10.3145/thinkepi.2019.e13e09

Díaz Nosty, B. y de Frutos, R. (Coords.) (2017). Tendencias de la investigación universitaria española en comunicación. Pamplona: Thomson Reuters / Aranzadi.

García García, F. (2007). Los medios de comunicación y los códigos de la UNESCO. Icono 5(1), 384-396. https://doi.org/10.7195/ri14.v5i1.383

García-Jiménez, L. (2016). No hay nada más práctico que una buena teoría: reflexiones a partir del metamodelo constitutivo de Rorbert T. Craig. En E. Vizer y C. Vidales (Coord.), Comunicación, campo(s), teorías y problemas (pp. 337-355). Salamanca: Comunicación Social, Ediciones y Publicaciones.

Giménez, E. (2016). Malestar. Los investigadores ante su evaluación. Madrid: Iberoamericana.

Goyanes, M. (2017). Desafío a la investigación estándar en comunicación. Crítica y alternativas. Barcelona: Editorial UOC.

Goyanes, M. y Rodríguez-Gómez, E. F. (2018). ¿Por qué publicamos? Prevalencia, motivaciones y consecuencias de publicar o perecer. El Profesional de la Información, 27(3), 548-558. https://doi.org/10.3145/epi.2018.may.08

Marzal-Felici, J., García-Jiménez, A. y Humanes, M. L. (2016). Análisis y reformulación de la organización del conocimiento en las Ciencias de la Comunicación: aplicación para la codificación UNESCO. Revista General de Información y Documentación, 26(1), 65-79. http://dx.doi.org/10.5209/rev_RGID.2016.v26.n1.53049

Marzal Felici, J., Rodríguez Serrano, A. y Gil Soldevilla, S. (2018). Introducción: la investigación en la era de la expansión del pensamiento neoliberal. Reflexiones sobre la naturaleza de la investigación en comunicación. En: A. Rodríguez Serrano y S. Gil 
Soldevilla (Eds.), Investigar en la era neoliberal. Visiones críticas sobre la investigación en comunicación en España (pp. 13-26). Barcelona, Castellón y Valencia: Colección Aldea Global, Servicios de Publicaciones de la UAB, UJI, UPF y UV.

Marzal-Felici, J., Soler-Campillo, M. y Rodríguez-Serrano, A. (2020). Los estudios reglados de comunicación en las universidades del ranking de Shangai. Propuestas para una epistemología de las ciencias de la comunicación. Revista Mediterránea de Comunicación/Mediterranean Journal of Communication, 11(1), 179-194. https://www.doi.org/10.14198/MEDCOM2020.11.1.17

Ministerio de Economía y Competitividad (2012). Estrategia española de Ciencias y Tecnología y de Innovación. Madrid: Ministerio de Economía y Competitividad. Recuperado de https://bit.ly/2UzAXEV

Muller, J. Z. (2018). The Tyranny of Metrics. Princeton, New Jersey: Princeton University Press.

Orduna-Malea, E., Martín-Martín, A., Delgado López-Cózar, E. (2017). Métricas en perfiles académicos: ¿un nuevo juego adictivo para los investigadores? Revista Española de Salud Pública, 90, e20006. Recuperado de https://www.ncbi.nlm.nih.gov/pubmed/27653216

Piñuel, J. L.; Sánchez Carrión, J. J.; Peñafiel, C.; Marzal Felici, J. (2017). El estudio MapCom y la investigación en España en las tesis doctorales y los proyectos de I+D. En B. Díaz Nosty y R. de Frutos (Coords.), Tendencias de la investigación universitaria española en comunicación (pp. 13-33). Pamplona: Thomson Reuters / Aranzadi.

Pels, D. (2003). Unhastening Science: Autonomy and Reflexivity in the Social Theory of Knowledge. Liverpool: Liverpool University Press.

Reig, R. (2014). La investigación dependiente: crítica estructural al sistema JCR. Revista Ámbitos, no 27, cuarto trimestre (invierno). Recuperado de https://idus.us.es/handle/11441/66501 